\title{
BLACK HOLES IN 4 NEARBY RADIO GALAXIES
}

\author{
Jeremy Mould ${ }^{1}$, Tony Readhead ${ }^{3}$, Garret Cotter ${ }^{4}$, David Batt ${ }^{2}$ and Mark Durré ${ }^{1}$ \\ jmould@swin.edu. au
}

Received __; accepted _

\footnotetext{
1 Centre for Astrophysics and Supercomputing, Swinburne University of Technology, Melbourne, Victoria 3122, Australia

2 School of Physics, University of Melbourne, Parkville, Victoria 3010, Australia

${ }^{3}$ California Institute of Technology, CA91125, USA

${ }^{4}$ Department of Physics, University of Oxford, Denys, UK
} 


\begin{abstract}
We study the velocity dispersion profiles of the nuclei of NGC 1326, 2685, 5273 and 5838 in the CO first overtone band. There is evidence for a black hole $(\mathrm{BH})$ in NGC 1326 and 5838. Gas is seen flowing out of the nuclear region of NGC 5273. We put upper limits on the nuclear BHs responsible for its activity and that of NGC 2685.
\end{abstract}

Subject headings: infrared: general — active galactic nuclei - galaxies: elliptical radiosources - black holes 


\section{Introduction}

Understanding activity in galactic nuclei requires high spatial resolution. Kormendy \& Richstone (1995) have outlined the techniques for quantifying the supermassive black holes that power active galactic nuclei (AGN). Our strategy (Mould et al 2012) is good seeing infrared spectroscopy of AGN in a volume limited sample, followed by adaptive optics spectroscopy on large aperture telescopes. In this paper we present Palomar TripleSpec spectra of a number of nearby radiogalaxies of early type.

NGC 1326 is a ring barred S0 galaxy in the Fornax cluster with circumnuclear star formation (Buta et al 2000). Our second galaxy is a Hubble Atlas polar ring galaxy, an S0 Seyfert 2. Schinnerer \& Scoville (2002) detected four giant molecular cloud associations within the polar ring in NGC 2685 (the Helix) with of order $10^{7} \mathrm{M}_{\odot}$ of molecular hydrogen. Dust has been detected with Spitzer in our third S0 galaxy, NGC 5273, totalling $2.5 \times 10^{5}$ $\mathrm{M}_{\odot}$ by Martini et al (2013). NGC5838 has a nuclear star cluster of $5 \times 10^{7} \mathrm{M}_{\odot}($ Scott \& Graham 2013).

\section{Sample and observations}

We have drawn our radiogalaxy sample from Brown et al (2011), further limiting the distance to $20 \mathrm{Mpc}$ in order to have $100 \mathrm{pc}$ resolution in $1^{\prime \prime}$ seeing. Observations of NGC 1326, 2685, 5273 \& 5838 were obtained on the Hale Telescope in 2011 and 2012. Obtaining

our Palomar TripleSpec spectra was described by Mould et al (2012) and data reduction was outlined by Batt et al (2014, Paper I). We very briefly recap this here. The spectrograph has resolution of 2600 with a $1^{\prime \prime}$ slit, and observations were made with the nucleus in two slit positions ABBA in $4 \times 5$ minutes. These were followed by observations of an A0 star for telluric correction and seeing measurement. Flatfielded spectra were subtracted and 
extracted at different impact parameters along the slit, yielding the wavelength shifts and first overtone CO line widths given in Table 1. The IRAF cross-correlation task $f x c o r$ was used for this purpose with the Gemini library stellar template HD2490 interpolated to the same resolution.

Table 1 gives the radial position of the extracted spectrum in column (1), the pixel shift between that and the template in column (2), the peak height of the cross-correlation in column (3) and the FWHM of the fit to the cross-correlation in column (4). The units of columns (2-4) are pixels. 
Table 1. Raw crosscorrelation data

\section{NGC 2685}

$\begin{array}{llllllll}\text { position } & \text { pixel shift } & \text { peak } & \text { fwhm } & \text { position } & \text { pixel shift } & \text { peak } & \text { fwhm } \\ (\operatorname{arcsec}) & {[2]} & {[3]} & {[4]} & (\operatorname{arcsec}) & {[2]} & {[3]} & {[4]} \\ 0 & -189.96 & 0.23 & 15.4 & 0 & -190.55 & 0.21 & 12.16 \\ 0 & -190.68 & 0.26 & 14.96 & 0 & -190.7 & 0.25 & 15.1 \\ 0.9 & -190.5 & 0.26 & 14.2 & 1.63 & -187.09 & 0.24 & 14.8 \\ 0.73 & -188.4 & 0.27 & 13.9 & 0.79 & -188.99 & 0.18 & 14.98 \\ 0.79 & -190.65 & 0.26 & 13.41 & 0.84 & -189.96 & 0.2 & 12.7 \\ 0.79 & -191.01 & 0.2 & 12.3 & & & & \end{array}$

\section{NGC 5838}

$\begin{array}{llllllll}\text { position } & \text { peak height } & \text { fwhm } & \text { shift } & \text { position } & \text { peak height } & \text { fwhm } & \text { shift } \\ (\operatorname{arcsec}) & {[2]} & {[3]} & {[4]} & (\operatorname{arcsec}) & {[2]} & {[3]} & {[4]} \\ 0 & 0.366 & 27.4 & -0.76 & 0 & 0.519 & 29.1 & -0.67 \\ 0.316 & 0.329 & 27.1 & -1.31 & 0.632 & 0.301 & 13.1 & -0.1 \\ 0.948 & 0.333 & 14.2 & -0.6 & 1.264 & 0.368 & 10.2 & -0.41 \\ 1.58 & 0.331 & 10 & -0.27 & 1.896 & 0.314 & 8.18 & -0.08 \\ 2.212 & 0.322 & 11.4 & -0.28 & 0.316 & 0.325 & 13.1 & 0.55 \\ 0.632 & 0.391 & 8.35 & -0.03 & 0.948 & 0.398 & 8.99 & 0.249 \\ 1.264 & 0.476 & 10.6 & 0.01 & 1.58 & 0.462 & 12.3 & 0.4 \\ 1.896 & 0.484 & 18.6 & 0.89 & 2.212 & 0.503 & 14.3 & 1.01 \\ 0.316 & 0.397 & 13.7 & -0.13 & 0.632 & 0.398 & 14.6 & -0.4\end{array}$


Table 1-Continued

\begin{tabular}{llllll}
\hline \hline & & & & & \\
& & & & & \\
& & & & & \\
0.948 & 0.394 & 11.7 & -0.71 & 1.264 & 0.39 \\
1.58 & 0.418 & 8.8 & -0.47 & 1.896 & 0.381 \\
& & & & & \\
NGC 5273 & & & & & \\
position & peak & fwhm & position & peak & fwhm \\
$($ arcsec) & {$[2]$} & {$[3]$} & $($ arcsec $)$ & {$[2]$} & {$[3]$} \\
0 & 0.28 & 28.36 & 0 & 0.22 & 15.6 \\
0 & 0.28 & 23.22 & 0 & 0.22 & 20.63 \\
1.57 & 0.13 & 21.09 & 0.73 & 0.26 & 17.12 \\
1.99 & 0.23 & 16.76 & 1.09 & 0.23 & 24.18 \\
1.58 & 0.12 & 11.94 & 0.79 & 0.28 & 20.57 \\
1.69 & 0.12 & 14.22 & 0.9 & 0.2 & 11.31 \\
1.18 & 0.18 & 21.12 & 1.97 & 0.16 & 7.22 \\
0.54 & 0.19 & 22.96 & 1.33 & 0.1 & 21.68 \\
0.84 & 0.29 & 27.98 & 1.63 & 0.13 & 15.2 \\
1.07 & 24.97 & 1.86 & 0.19 & 14.78
\end{tabular}

NGC 1326

$\begin{array}{llllll}\text { position } & \text { peak } & \text { fwhm } & \text { position } & \text { peak } & \text { fwhm } \\ 0 & 0.4 & 20.61 & 0 & 0.39 & 20.56 \\ 0 & 0.4 & 20.13 & 0 & 0.42 & 20.84 \\ 0.78 & 0.43 & 22.4 & 0.56 & 0.41 & 21.94\end{array}$




\section{Kinematics and dynamics}

\subsection{NGC 1326}

NGC 1326 has been imaged by the Hubble Space Telescope (Figure 1) and its ultraviolet light distribution is displayed in the radial profile from the IRAF STSDAS surface photometry task ellipse in Figure 2.

The Jeans equation allows us to predict the velocity dispersion profile $\sigma(\mathrm{r})$ corresponding to this light distribution, assuming spherically distributed stars on isotropic orbits. To do this, we need the logarithmic derivatives with respect to radius of the density and velocity dispersion profiles. The former is obtained numerically using an Abell transform, the latter by calculating the (small) slope of the velocity dispersion data. The visual mass to light ratio is a free parameter in this model and we fit it to the data at $\mathrm{r}>80 \mathrm{pc}$, finding $\mathrm{M} / \mathrm{L}$ $=6.5$ in solar units, a normal value for a stellar population not dominated by dark matter. TripleSpec line width values were normalized in the same way as in Paper I.

The addition of a $1 \times 10^{7} M_{\odot}$ black hole modifies the mass distribution and $\sigma(\mathrm{r})$. It is a better fit to the data than the solid line in the lower part of Figure 2. The no BH model is ruled out with $70 \%$ confidence based on $\chi^{2}$.

\subsection{NGC 2685}

NGC 2685 is a polar ring galaxy, known as 'the spindle'. The HST nuclear image is reproduced in Figure 3 and the light distribution has been fitted with a 'nuker profile' (Lauer et al 2007). The profile appears in Figure 4, the model fit, and $\chi^{2}$ per degree of

freedom implies that $\mathrm{M}_{\bullet}>3 \times 10^{7}$ with less than $20 \%$ probability. This is consistent with Beifiori et al (2009), who find an upper limit $\mathrm{M}_{\bullet}<1.1 \times 10^{7} M_{\odot}$. The innermost datapoint 
has been located, not at zero radius as Table 1 would imply, but at the effective light centre of the zero radius observation taking account of seeing.

\subsection{NGC 5273}

We fitted a nuker profile to archival HST WFPC2 PC data (Figure 5), obtaining $(\alpha, \beta, \gamma)=(1.8,1.8,0.75)$ and normalized the profile to the surface photometry of Muñoz

Marin et al (2007) with $\mathrm{r}_{b}=50$ pc. Figure 6 is the model fit, and $\chi^{2}$ per degree of freedom implies that $\mathrm{M}_{\bullet}>10^{8}$ with less than $25 \%$ probability. We assumed the Tonry et al (2001) surface brightness fluctuations distance of $\mathrm{m}-\mathrm{M}=31.09 \pm 0.26$.

The TripleSpec spectrum also shows an interesting He I 10830§ line (Figure 10). Silhouetted against the broad line region helium emission and its luminous $\left(10^{8} \mathrm{~L}_{\odot}\right)$ x-ray gas (Liu 2011) is a P-Cyg profile of cooler ( $\mathrm{kT} \sim 30 \mathrm{eV})$ neutral gas with a terminal outflow velocity of $750 \mathrm{~km} / \mathrm{sec}$. This object will repay IFU study of its circumnuclear gas and modelling to determine the outflow rate.

\subsection{NGC 5838}

Calculation of a predicted stellar velocity dispersion profile was described for galaxies with nuker profiles in Paper I. NGC 5838 has such a profile (Lauer et al 2007). Figure 7 shows the nucleus of NGC 5838 and Figure 8 shows a fit with $\mathrm{M} / \mathrm{L}=30$ and a black hole of $1 \times 10^{8} \mathrm{M}_{\odot}$. Note that Lauer et al assume $\mathrm{V}-\mathrm{H}=2.39$ in converting NICMOS data to visual magnitudes. We also adopted their distance of $22.2 \mathrm{Mpc}$. The no $\mathrm{BH}$ model is rejected with $98 \%$ confidence. 


\section{Summary}

We summarize our findings in Table 2. In two cases we have SMBH detections; in two cases we have upper limits on the SMBH mass. Our upper limit for NGC 5273 is consistent with the result from reverberation mapping of $4.7 \pm 1.6 \times 10^{6} M_{\odot}$ by Bentz et al 2014 . Figure 9 shows our 4 radio galaxies in their Magorrian diagram. NGC 5838 is plotted at $\sigma$ $=290 \mathrm{~km} / \mathrm{sec}$ (McElroy 1995).

\section{Table 2: Black hole masses}

\begin{tabular}{|c|c|c|c|c|c|}
\hline \multirow{2}{*}{$\begin{array}{l}\text { Name } \\
\text { NGC }\end{array}$} & Type & Distan & $\mathrm{M}_{V}$ & \multicolumn{2}{|c|}{ M/L SMBH } \\
\hline & & $(\mathrm{Mpc})$ & & & $\mathrm{M}_{\odot}$ \\
\hline N1326 & SB0+ & 20.5 & -21.05 & 6.5 & $1 \times 10^{7}$ \\
\hline N2685 & SB0+ & 14.3 & -19.72 & 1.3 & $<3^{*} \times 10^{7}$ \\
\hline N5273 & So & 20 & -20.1 & $1^{\dagger}$ & $<1 \times 10^{8}$ \\
\hline 5838 & SO- & 22.2 & -20.51 & 30 & $1 \times 10^{8}$ \\
\hline
\end{tabular}

We thank our referee for comments that improved the paper. We are grateful for the support of the Australian Research Council through DP140100435. GC acknowledges support from STFC grant ST/K005596/1. Spectra were extracted using a version of the Spextool program modified for the Palomar TripleSpec Spectrograph (Cushing et al. 2004, ; M. Cushing, private communication 2011). We acknowledge the Hubble Legacy Archive, a facility of STScI, which is operated by AURA for the National Aeronautics and Space Administration (NASA). This research has made use of the NASA/IPAC Extragalactic 
Database (NED) which is operated by the Jet Propulsion Laboratory, California Institute of Technology, under contract with NASA. This research has also made use of IRAF, software written by NOAO and data products from the Gemini Observatory, which are operated by AURA under a cooperative agreement with NSF. David Batt was a summer student at Swinburne University while this work was carried out. 
Table 1-Continued

\begin{tabular}{llllll}
\hline \hline & & & & & \\
& & & & & \\
\hline & & & & & \\
1.62 & 0.43 & 22.17 & 1.12 & 0.38 & 20.38 \\
1.01 & 0.42 & 22.8 & 0.95 & 0.43 & 21.99 \\
1.57 & 0.41 & 22.61 & 1.8 & 0.39 & 24.8 \\
0.45 & 0.42 & 21.96 & 0.62 & 0.44 & 21.96 \\
1.41 & 0.41 & 20.65 & 1.07 & 0.42 & 21.61 \\
0.9 & 0.41 & 20.88 & 0.68 & 0.42 & 21.4 \\
1.52 & 0.39 & 20.5 & 1.52 & 0.41 & 22.17 \\
\hline
\end{tabular}




\section{REFERENCES}

Batt, D. et al 2014, in prep.

Bentz, M. et al 2014, ApJ, in press, astro-ph 1409.5794

Buta, R. 2000, AJ, 120, 1289

Beifiori, A. et al 2009, ApJ, 692, 856

Brown, M. et al 2011, ApJ, 731, L41

Cushing, M., Vacca, W., \& Rayner, J. 2004, PASP, 116, 362

Durré, M. \& Mould, J. 2014, ApJ, 784, 79

Kormendy \& Richstone 1995, ARAA, 33, 581

Lauer, T. et al 2007, ApJ, 664, 226

Liu, J. 2011, ApJS, 192,10

Martini, P. et al 2013, ApJ, 766, 121

McElroy, D. 1995, ApJS, 100, 105

Mould, J. et al 2012, ApJS, 203, 14

Muñoz Marín, V. et al 2007, AJ, 134, 648

Schinnerer, E. \& Scoville, N. 2002, ApJ, 577, L103

Scott, N. \& Graham, A. 2013, ApJ, 763, 76

Tonry, J. et al 2001, ApJ, 546, 681 


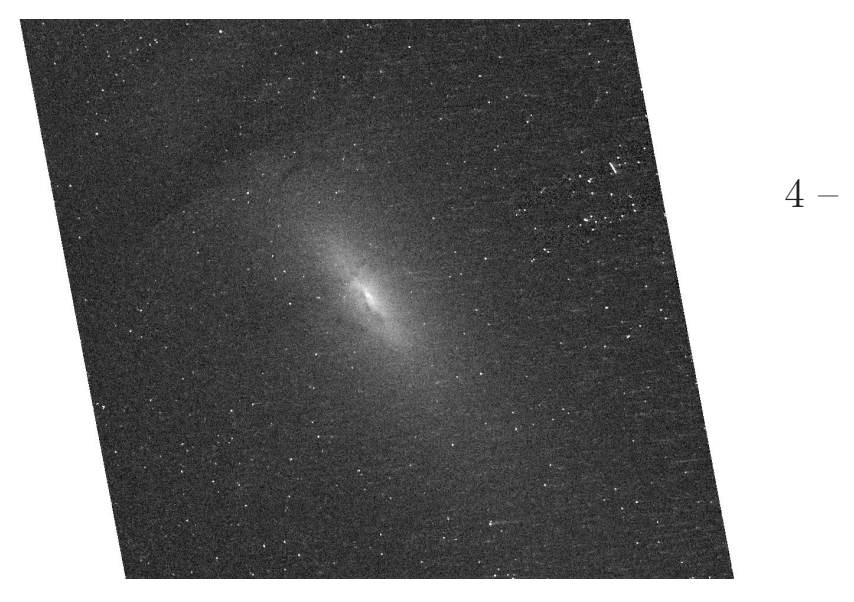

Fig. 3.- The HST ACS HRC image of the nucleus of NGC2685. The filter is F330W.

NGC2685
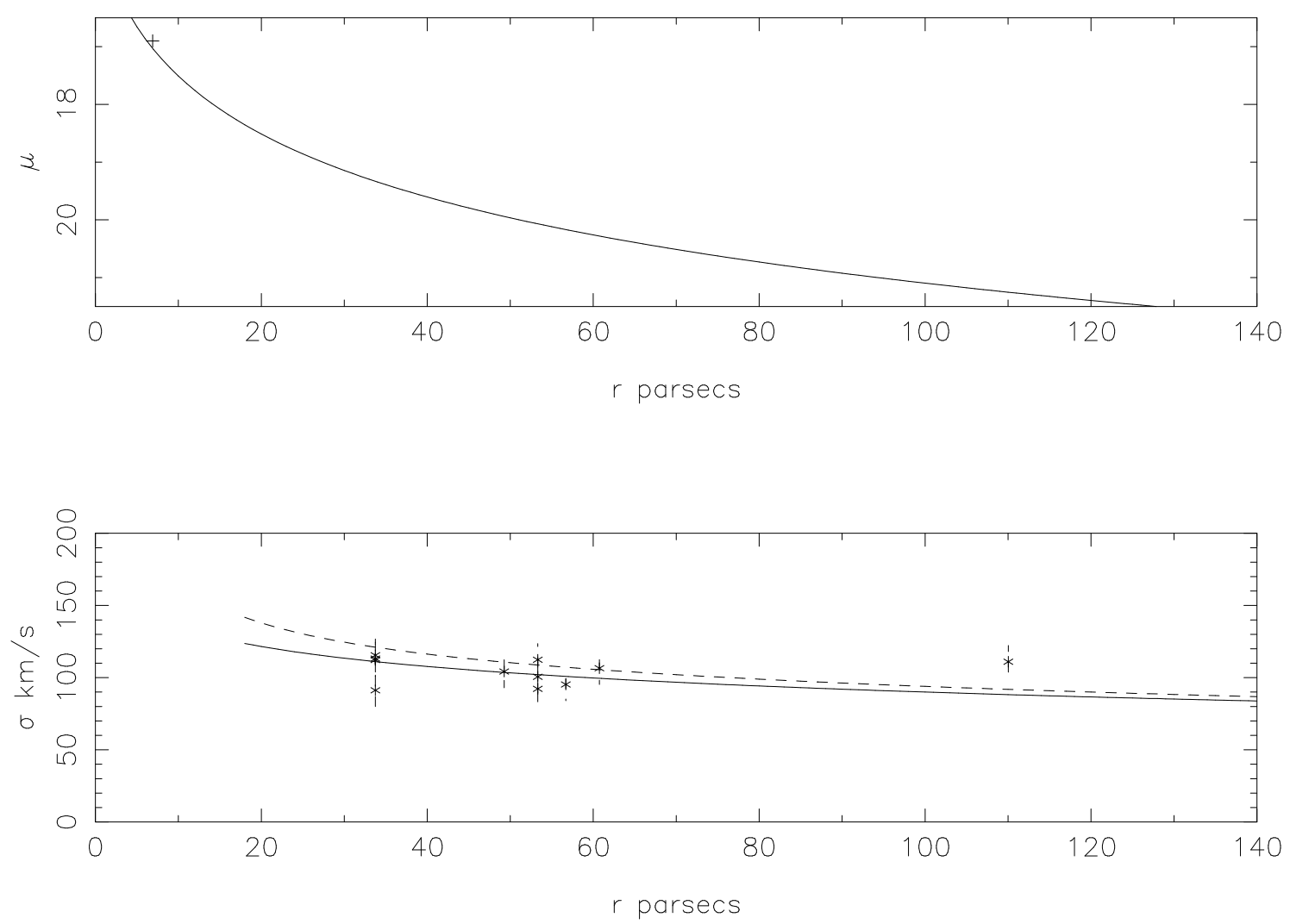

Fig. 4.- top NGC 2685's nuker profile; bottom a fit to the TripleSpec data without and with (dashed line) a $1.2 \times 10^{7} \mathrm{M}_{\odot} \mathrm{SMBH}$. 


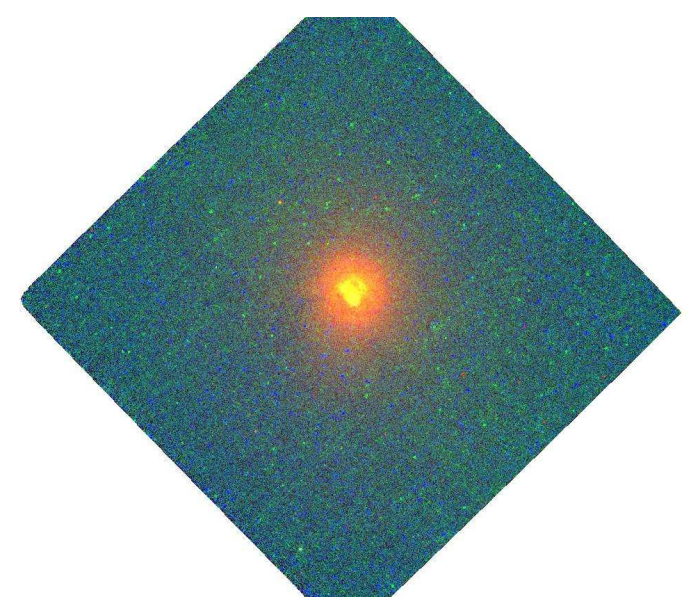

Fig. 5. - The HST WFPC2 PC image of the nucleus of NGC5273. The filters in RGB order are F547M/F300W/F218W. 
NGC5273
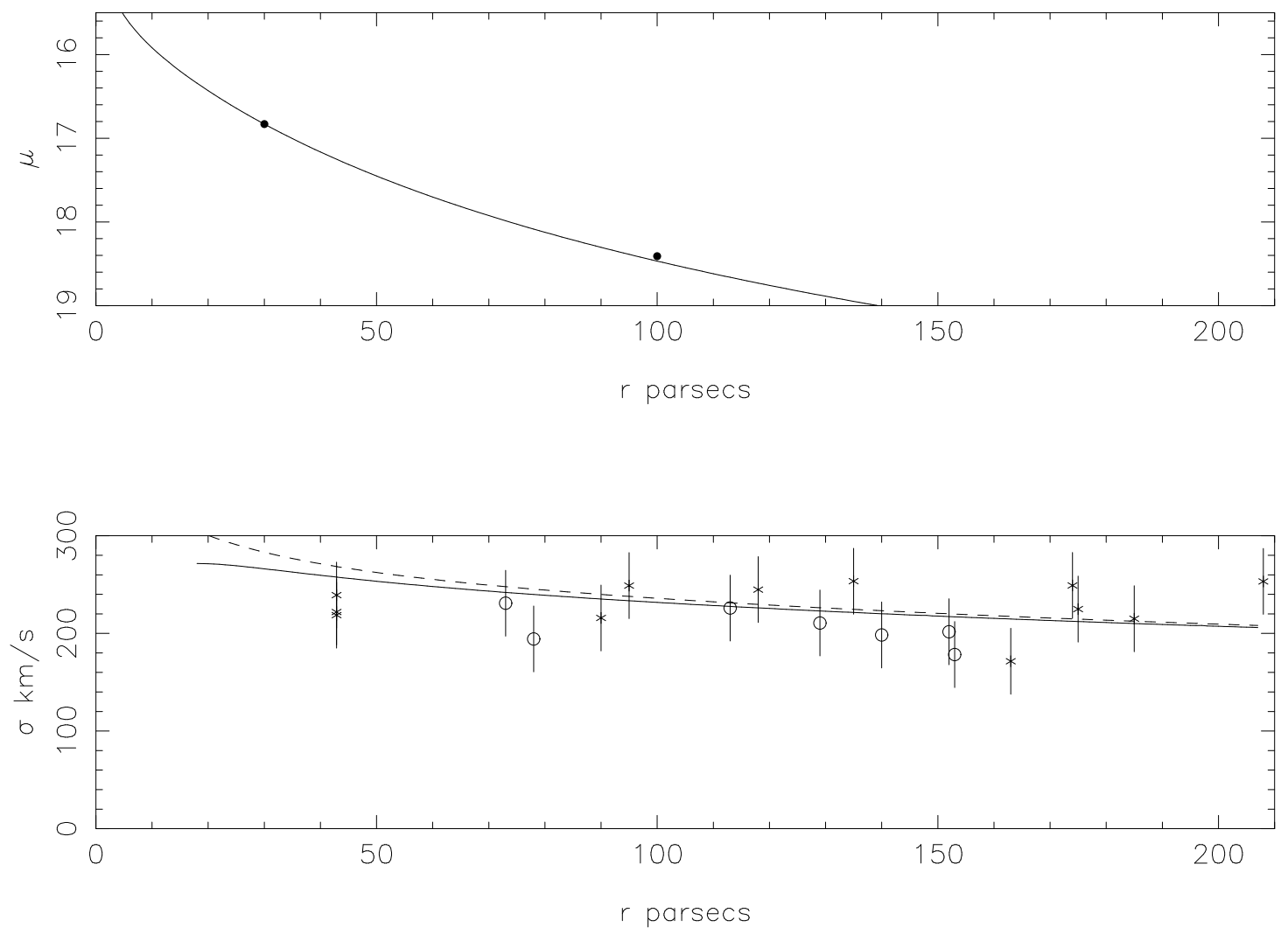

Fig. 6. - top the two data points are from Muñoz Marin et al 2007; bottom the dashed line has a $\mathrm{SMBH}$ of $1 \times 10^{8} \mathrm{M}_{\odot}$. Error bars are proportional to the cross correlation peak heights in Table 1. 


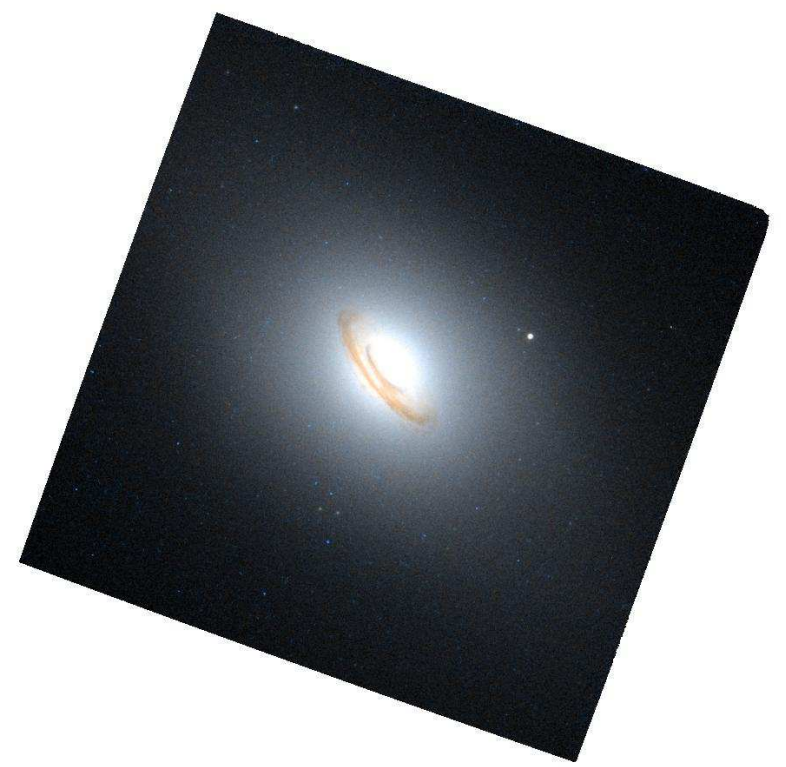

Fig. 7.- The HST WFPC2 PC image of the nucleus of NGC5838. The blue and red filters are F450W and F814W. NASA: Hubble Legacy Archive.

NGC5838
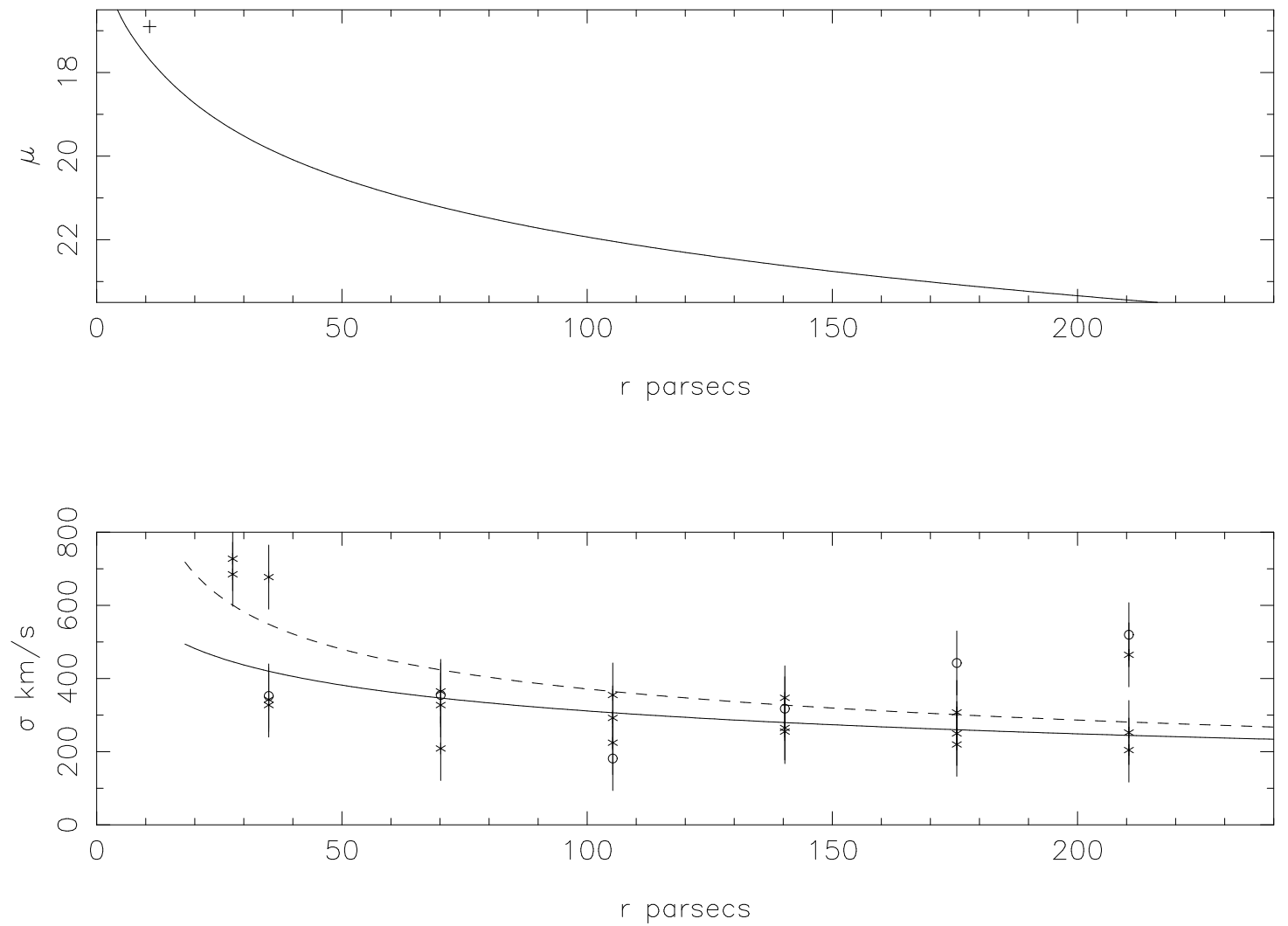

Fig. 8. - The lower figure compares a fit to NGC 5838 with M/L = 30 (solid line) and adding a SMBH with mass $1 \times 10^{8} \mathrm{M}_{\odot}$ (dashed line). 


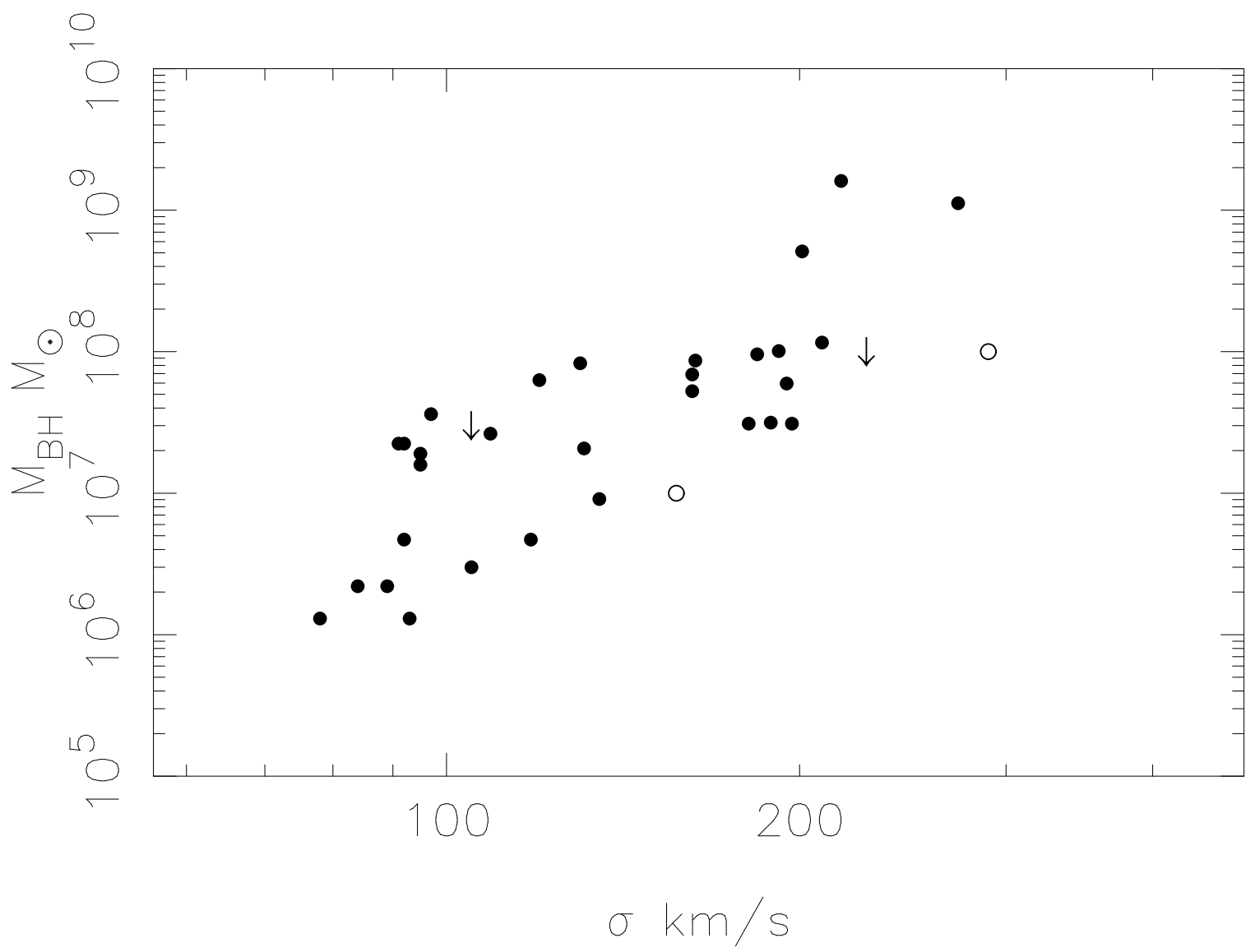

Fig. 9.- Our two detections and two upper limits in the Magorrian diagram of Bentz et al (2014). 


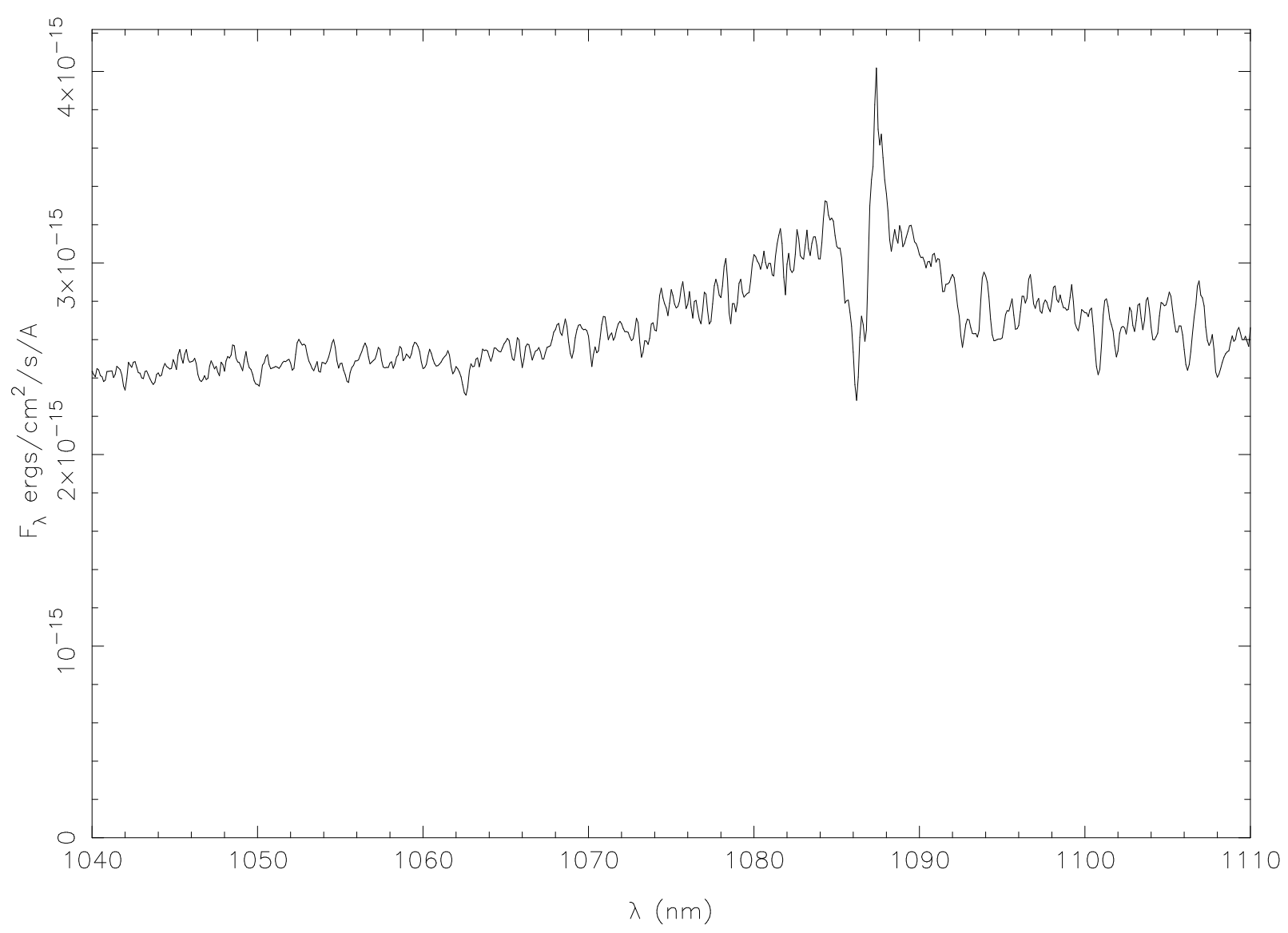

Fig. 10. - The He I $10830 \AA$ region of NGC 5273. 